ORIGINAL ARTICLE

\title{
Prognostic relevance of extensive necrosis in renal cell carcinoma
}

\author{
V Foria, T Surendra, D N Poller
}

J Clin Pathol 2005;58:39-43. doi: 10.1136/jicp.2004.018846

\begin{abstract}
See end of article for authors' affiliations

....................

Correspondence to: Dr D N Poller, Department of Pathology, Queen Alexandra Hospital Cosham, Portsmouth PO6 3LY, UK; david.poller@ porthosp.nhs.uk
\end{abstract}

Accepted for publication 14 June 2004

\begin{abstract}
Aims: Evidence suggests that the presence of tumour necrosis is an adverse prognostic factor in renal cell carcinoma (RCC). However, it has also been shown that tumour regression, a microscopic feature associated with necrosis, may be a favourable short term prognostic factor in RCC.

Methods: Pathology reports of 253 RCCs from 1992 to 2001 were reviewed, and identified 37 tumours with substantial macroscopic or microscopic necrosis. Microscopic pathology, TNM 1997 tumour stage, and clinical follow up were reviewed and correlated with pathological findings. Three cases were rejected because two were diagnosed at necropsy, and a third was the result of renal arterial embolisation.

Results: Twenty of the 34 cases showed $<50 \%$ necrosis, 10 showed $50-94 \%$ tumour necrosis, and four showed $>95 \%$ tumour necrosis. Follow up data were unavailable in three cases. Nine of the remaining 31 tumours progressed; six were group 3 tumours showing $<50 \%$ necrosis, three were group 2 tumours showing $50-94 \%$ necrosis, and none was a group 1 tumour showing $>95 \%$ necrosis.

Conclusions: Extensive necrosis (>95\% necrosis) is rare in RCC, accounting for only $1.6 \%$ of those diagnosed during eight years in this unselected hospital series. The microscopic pattern of necrosis was typical, requiring extensive tumour sampling for definitive tumour diagnosis. Although there were only four patients with extensive necrosis, none developed recurrent or metastatic carcinoma, or died from RCC. Although extensive (>95\%) necrosis may imply better short term prognosis after adjusting for tumour pathological TNM stage, it is probably not a prognostic variable in RCC.
\end{abstract}

1 mportant prognostic factors in renal cell carcinoma (RCC) include tumour subtype, ${ }^{12}$ tumour TNM stage, ${ }^{3-5}$ nuclear grade, ${ }^{1256}$ the presence of a sarcomatoid component, ${ }^{12}$ and any evidence of tumour necrosis. ${ }^{125-8}$ However, the importance of extensive necrosis, which is rare in RCC, is not entirely clear. ${ }^{910}$ A recent study suggested that RCC with very extensive necrosis may be capable of aggressive behaviour. ${ }^{8}$ Necrosis is commonly seen in RCC and necrosis has been shown to be an adverse prognostic factor in certain subtypes of RCC, if any necrosis at all is present in the tumour sections. ${ }^{125-8}$

The pathology reports of all cases of RCC diagnosed over an eight year period were retrieved from our laboratory files to identify tumours showing extensive necrosis. The clinical follow up data of these patients were then obtained.

\section{MATERIALS AND METHODS}

The pathology reports of all 253 RCCs diagnosed between 1992 and 2001 at the department of pathology at Queen Alexandra Hospital, Portsmouth, UK, were reviewed after they were identified by searching the laboratory computer database using a SNOMED search for all RCCs. We identified 37 tumours where the pathologist had commented that there was evidence of either macroscopic or microscopic necrosis at the time of originally reporting the case. The gross descriptions and all available slides from these 37 cases were then reviewed in detail, without prior knowledge of the clinical follow up data or patient prognostic information. The overall percentage of necrosis in each case was assessed from all the available haematoxylin and eosin stained slides of each tumour. The cases were categorised into three groups, namely: group 1, more than $95 \%$ necrosis within the cross sectional area of available sections of tumour; group 2, 50$94 \%$ necrosis within the available sections of tumour; and group 3, less than $50 \%$ necrosis within the available tumour sections. Tumours with evidence of tumour hyalinisation were also included and scored as necrotic non-viable tumours, as were those that showed evidence of cystic change. The tumour nuclei were graded according to the Fuhrman system. ${ }^{11}$ The age and sex of the patients were recorded from the pathology report, along with the maximum macroscopic size of the tumour. Tumours were staged using the data in the pathology reports and review of the slides, according to TNM 1997. ${ }^{12}$ The case notes were consulted for the follow up data. Progression of disease was defined as either regional recurrence, development of metastasis, or death as a result of disease. Two cases with extensive necrosis $(>95 \%)$ were rejected because these were unexpected incidental findings at necropsy in patients dying of intercurrent disease. A third case that showed extensive histological necrosis arose as a result of tumour necrosis and infarction caused by therapeutic renal arterial embolisation, and is not further discussed.

\section{RESULTS}

Of the remaining 34 cases, four showed more than $95 \%$ necrosis, 10 cases showed 50-94\% necrosis, and 20 cases showed less than $50 \%$ necrosis (table 1). The pattern of necrosis in all four cases showing extensive ( $>95 \%$ ) necrosis was similar. There was a central necrotic core consisting of cellular debris, often with cholesterol clefts and haemorrhage. Around this was a thin rim of viable tumour cells surrounded by a thick fibrous capsule. The tumours required extensive tissue sampling, sometimes with immunohistochemistry for cytokeratins, to make a confident diagnosis of malignancy. Some extensively necrotic (>95\% necrotic) tumours from group 1 showed cystic areas lined by flattened attenuated epithelium. The necrotic centres of the tumours either contained amorphous material, with scattered calcifications, with sometimes cholesterol clefts and macrophage infiltration of the necrotic central area of these tumours. Other cases showed central tumour hyalinisation and partial 
Table 1 Clinicopathological data of all cases

\begin{tabular}{|c|c|c|c|c|c|c|c|}
\hline Group & $\begin{array}{l}\text { No of } \\
\text { cases }\end{array}$ & $\begin{array}{l}\text { Mean (range) } \\
\text { age (years) }\end{array}$ & $\begin{array}{l}\text { Sex } \\
\text { (M/F) }\end{array}$ & $\begin{array}{l}\text { Mean (range) tumour } \\
\text { size }(\mathrm{mm})\end{array}$ & TNM stage & Grade & Progression \\
\hline Group 1 & 4 & $58(27-82)$ & $3 / 1$ & $80.2(40-180)$ & $\begin{array}{l}\text { I: } 3 \\
\text { II: } 1 \\
\text { III: } 0 \\
\text { IV: } 0\end{array}$ & $\begin{array}{l}1: 2 \\
2: 2 \\
\text { 3: } 0 \\
4: 0\end{array}$ & $\begin{array}{l}0 / 4 \\
0 / 4\end{array}$ \\
\hline Group 2 & 10 & $63(32-79)$ & $7 / 3$ & $103.5(28-270)$ & $\begin{array}{l}\text { I: } 2 \\
\text { II: } 3 \\
\text { III: } 4 \\
\text { IV: } 0 \\
\text { NS: } 1\end{array}$ & $\begin{array}{l}1: 1 \\
2: 5 \\
3: 5 \\
4: 0\end{array}$ & $3 / 9(0,22,50)$ and 1 LTFU \\
\hline Group 3 & 20 & $62(44-80)$ & $13 / 7$ & $106.1(25-350)$ & $\begin{array}{l}\text { I: } 6 \\
\text { II: } 4 \\
\text { III: } 10 \\
\text { IV: } 0\end{array}$ & $\begin{array}{l}1: 2 \\
2: 15 \\
\text { 3: } 3 \\
4: 0\end{array}$ & $6 / 18(1,5,15,16,31,33)$ and 2 LTFU \\
\hline
\end{tabular}

Group 1, >95\% tumour necrosis; group 2, 50-94\% tumour necrosis; group 3, <50\% tumour necrosis.

NS, not staged; LTFU, lost to follow up.

Table 2 Group 1 (>95\% tumour necrosis)

\begin{tabular}{lllllll}
\hline Age (years) & Sex & Maximum tumour size $(\mathbf{m m})$ & pTNM stage & Fuhrman nuclear grade & Tumour subtype & Follow up (months) \\
\hline 56 & $M$ & 41 & 1 & 2 & Clear cell \\
82 & $M$ & 180 & 2 & 2 & NED (51) & Papillary \\
27 & F & 40 & 1 & 1 & Clear cell & NED (27) \\
67 & $M$ & 60 & 1 & 1 & Clear cell & NED (23) \\
\hline
\end{tabular}

DOOC, died of other causes; NED, no evidence of disease.

Table 3 Group 2 (50-94\% tumour necrosis)

\begin{tabular}{|c|c|c|c|c|c|c|}
\hline Age (years) & Sex & $\begin{array}{l}\text { Maximum tumour } \\
\text { size }(\mathrm{mm})\end{array}$ & pTNM stage & $\begin{array}{l}\text { Fuhrman } \\
\text { nuclear grade }\end{array}$ & Tumour subtype & Follow up (months) \\
\hline 79 & $M$ & 140 & 2 & 2 & Clear cell & NED (12) \\
\hline 32 & M & 82 & 2 & 1 & Clear cell & NED (50) \\
\hline 77 & $\mathrm{~F}$ & 100 & $3 b$ & 3 & Clear cell & Died PO \\
\hline 41 & $\mathrm{~F}$ & 28 & 1 & 2 & Clear cell & NED (65) \\
\hline 65 & $M$ & 160 & 3 & 2 & Clear cell & DOOC (11) \\
\hline 68 & M & NR & Unknown & 3 & Clear cell & LTFU \\
\hline 56 & $\mathrm{~F}$ & 70 & 1 & 2 & Clear cell & NED (70) \\
\hline 78 & $M$ & 270 & 3 & 3 & Clear cell & Recurrence $(50)$ \\
\hline 72 & $M$ & 95 & $3 b$ & 3 & Clear cell & Died (22) \\
\hline 67 & $M$ & 120 & 2 & 2 & Papillary & NED (106) \\
\hline
\end{tabular}

DOOC, died of other causes; LTFU, lost to follow up; NED, no evidence of disease; PO, postoperatively.

tumour calcification, without the presence of necrotic debris, with a few remaining viable tumour cells surrounding the periphery of the lesion-for example, case 1 in table 2, the first case included in a previous report. ${ }^{10}$

The number of blocks examined for group 1 cases ranged from nine to 23 (mean, 14.7). The number of blocks examined for group 2 cases ranged from four to 10 (mean, five) and for group 3 cases from one to 19 (mean, six). All three tumour groups (tables 2, 3, and 4) showed a broad age range, with mean age at presentation in the 7 th decade. A striking male predominance was noted in all three groups $(\mathrm{M}: \mathrm{F}$ ratio, 2:1). Tumours ranged in size from 25 to $350 \mathrm{~mm}$, with groups 2 and 3 having larger mean sizes; however, no significant size difference was noted. Although the number of cases in our series is small, making statistical comparisons difficult, group 1 tumours were all pTl or pT2, whereas a proportion of group 2 and 3 tumours were pT2 or pT3. One group 2 tumour could not be staged because the macroscopic size of the tumour was not mentioned in the pathology report. Of the 14 tumours that were pT3, in only five cases was there invasion of the tumour into the renal vein. Of these, two patients were in group 2 and three patients were in group 3 . With regard to grading, most of the cases in all three categories were Fuhrman nuclear grade 2 (22 of 34 cases).

\section{Patient outcome}

Follow up data were available for 31 patients, because three patients were lost to follow up. In the 31 patients in the series with follow up data, nine patients progressed and six of these showed less than 50\% necrosis and three showed between $50 \%$ and $94 \%$ necrosis. Follow up information was available for 18 of 20 patients with TNM stage I or II tumours. No patients with TNM stage I or II tumours progressed.

\section{Group 1}

The follow up time for the group 1 patients ranged from 23 to 51 months, with a mean follow up time of 34 months. None of the pathological group 1 patients showed tumour progression (table 2). 


\begin{tabular}{|c|c|c|c|c|c|c|}
\hline $\begin{array}{l}\text { Age } \\
\text { (years) }\end{array}$ & Sex & $\begin{array}{l}\text { Maximum tumour } \\
\text { size }(\mathrm{mm})\end{array}$ & pTNM stage & $\begin{array}{l}\text { Fuhrman } \\
\text { nuclear grade }\end{array}$ & Tumour subtype & Follow up (months) \\
\hline 70 & $M$ & 240 & 3 & 2 & Clear cell & DOOC (3) \\
\hline 46 & $\mathrm{~F}$ & 85 & $3 b$ & 2 & Clear cell & NED (13) \\
\hline 58 & $\mathrm{~F}$ & 75 & 2 & 2 & Clear cell & LTFU \\
\hline 79 & M & 350 & 2 & 1 & Clear cell & NED (24) \\
\hline 63 & $\mathrm{~F}$ & 25 & 1 & 2 & Clear cell & NED (25) \\
\hline 45 & $\mathrm{~F}$ & 170 & 3 & 2 & Clear cell & Metastasis (15) \\
\hline 63 & $M$ & 70 & 1 & 2 & Clear cell & NED (21) \\
\hline 71 & M & 32 & 1 & 2 & Clear cell & NED (39) \\
\hline 68 & $M$ & 70 & 3 & 2 & Clear cell & NED (39) \\
\hline 61 & $M$ & 200 & 2 & 2 & Clear cell & NED (37) \\
\hline 64 & $M$ & 80 & $3 b$ & 1 & Clear cell & Metastasis (33) \\
\hline 67 & $M$ & 50 & $3 b$ & 2 & Clear Cell & Metastasis (5) \\
\hline 44 & M & 40 & 1 & 2 & Clear cell & LTFU \\
\hline 64 & $M$ & 150 & $3 a$ & 3 & Clear cell & Metastasis (16) \\
\hline 53 & $\mathrm{~F}$ & 65 & 1 & 2 & Clear cell & NED $(60)$ \\
\hline 44 & $M$ & 140 & 3 & 3 & Clear cell & NED (57) \\
\hline 80 & $\mathrm{~F}$ & 190 & 3 & 3 & Clear cell & Died $(<1)$ \\
\hline 47 & $\mathrm{~F}$ & 200 & 2 & 2 & Clear cell & NED (73) \\
\hline 73 & $M$ & 85 & 3 & 2 & Clear cell & Metastasis (31) \\
\hline 73 & M & 120 & 2 & 2 & Papillary & NED (47) \\
\hline
\end{tabular}

\section{Group 2}

There were two patients with group 2 lesions who progressed. The first died of disease at 22 months and the second patient developed recurrence of tumour at 50 months, both tumours were pT3, with one showing invasion of the renal vein (table 3 ). A third patient with renal vein invasion died postoperatively. The mean follow up time for the six group 2 patients who did not progress was 52 months.

\section{Group 3}

The six patients with group 3 lesions who progressed were all stage pT3, and two showed evidence of renal vein invasion (table 4). One patient died within one month and the remainder developed metastases at five, 15, 16, 31, and 33 months, respectively. The follow up time for the 12 group 3 patients who did not progress ranged from 13 to 73 months, with the mean being 36 months.

\section{DISCUSSION}

The prognostic importance of identifying any necrosis in RCC is now clearly understood. Early studies had suggested that necrosis in RCC predicted adverse prognosis. ${ }^{13}$ This has now been confirmed in larger modern series of RCC, where any evidence of necrosis has been shown to be an adverse prognostic factor ${ }^{125-7}$ that ranks with tumour type, nuclear grade, and TNM stage as an independent predictor of adverse prognosis in clear cell RCC and chromophobe RCC. ${ }^{2}$

Cystic RCC has been classified into four morphological patterns by Hartmann et al. ${ }^{14}$ One of the four subtypes, the so called cystic necrosis subtype, corresponds to our group 1 cases. Hartmann and colleagues ${ }^{14}$ described these tumours as showing cystic degeneration that was so extensive that the bulk of the tumour is fluid filled, with a usually thick and irregular wall, with the cystic space filled with necrotic debris and fresh or old haemorrhage. They also described three other patterns of growth in cystic RCC, namely: intrinsic cystic growth multiloculated, intrinsic cystic growth (unilocular), and RCC arising in a pre-existing simple cyst.

The largest study of very extensive necrosis in RCC was a recent institutional series by Brinker et al. ${ }^{9}$ These authors identified and compared 17 cases of RCC showing very extensive necrosis with 26 cases showing at least 50\% necrosis and other cases of cystic RCC. The definition of extensive necrosis used by Brinker et al was any RCC tumour showing at least $99 \%$ necrosis. Brinker et al commented that in their type 1 cases (those tumours with more than 99\% necrosis), initial sampling of the tumour failed to reveal viable tumour, and total embedding of the tumour was required to identify small foci of recognisable cancer. Viable tumour was often only seen in the cyst walls, comprising small nests of clear cells, or in diminutive papillary structures, dilated tubular structures, or as papillary fronds projecting into a cystic cavity filled with necrotic debris. Brinker et al noted that the cystic nature of these lesions resulted from central tumour necrosis with a cyst content of calcific material and amorphous debris, with the cysts being surrounded by a thick fibrous capsule. Follow up information was available in $80 \%$ of their series. Nine cases progressed, although only one of these had more than $99 \%$ necrosis. ${ }^{9}$ The other eight cases that progressed showed a lesser degree of necrosis-greater than $50 \%$ but less than $99 \%$. Brinker et al

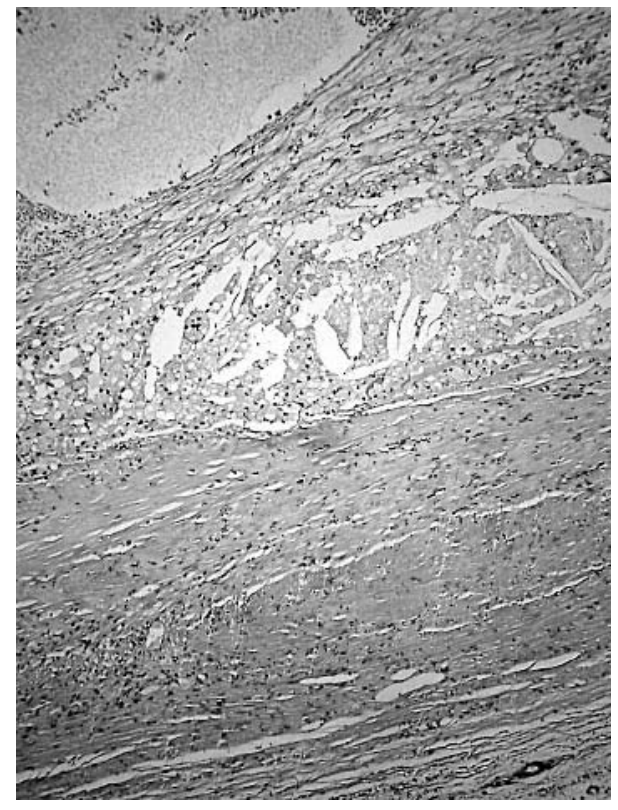

Figure 1 Typical appearances of a group 1 case showing more than $95 \%$ necrosis. The necrotic cystic cavity contains numerous histiocytes (top), with a barely visible attenuated epithelial lining (middle), surrounded by a thick fibrous capsule (bottom). 


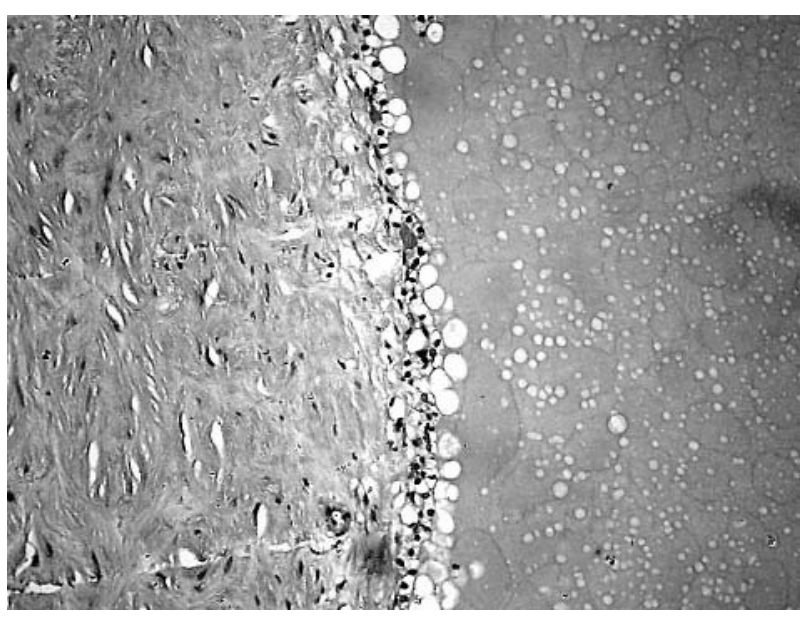

Figure 2 Another area from the same case shown in fig 1 in which the epithelial lining is more evident, with a thick fibrous tumour capsule.

concluded that RCC showing extensive necrosis is capable of aggressive behaviour and patients with these lesions cannot be assured of cure after surgery, although the short term prognosis of these tumours appeared to be relatively good. ${ }^{9}$

Two cases of extensively necrotic RCC included in our series were reported in $1998,{ }^{10}$ although without clinical follow up information. These two cases are group l, cases 1 and 2 (table 2 ). The previous report suggested that extensive tumour necrosis might have arisen as a result of spontaneous regression of $\mathrm{RCC}^{10}$ and emphasised, as did Brinker et al, ${ }^{9}$ the importance of adequate tissue sampling of the whole tumour to make a confident diagnosis of RCC. We have now identified from our laboratory's files two further cases of RCC showing extensive ( $>95 \%$ ) tumour necrosis. This total of four cases was compared and contrasted with a much larger number of RCCs showing lesser degrees of necrosis: group 2 showing 94-50\% necrosis and group 3 showing less than $50 \%$ tumour necrosis in tissue sections. In addition, two further cases of RCC were identified that showed extensive $(>95 \%)$ necrosis as coincidental findings at medicolegal necropsy, in patients dying of other diseases, but were not included in our series because there was no clinical follow up information available.

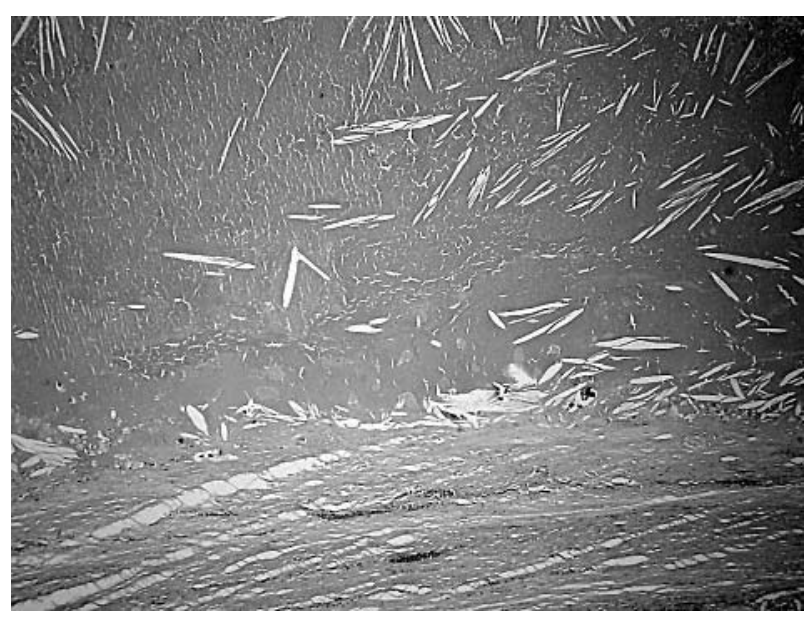

Figure 3 Different group 1 case showing the haemorrhagic contents of the cyst cavity with numerous cholesterol clefts (top) and a thick fibrous capsule (bottom).



Figure 4 Low power view of a group 1 case showing extensive central hyalinisation, with only a few viable tumour cells at the tumour centre. The hyalinised tumour area is seen at the top, with the surrounding normal renal cortex at the bottom. The normal renal tubules show membrane expression of Cam 5.2 low molecular weight cytokeratin.

Our study was undertaken to ascertain the prognostic relevance of extensive necrosis in RCC and to assess the prevalence of such cases in routine practice. All of the patients included in our study underwent surgery for RCC in our hospital, making estimation of the prevalence of extensive necrosis in RCC easier, because there is less likelihood of case selection bias that may be evident in large series derived from a pathology case referral practice. Inevitably, there is an element of subjectivity in assessing the degree of necrosis because the assessment of the degree of necrosis is based on the retrospective review of archival tissue sections.

In our study, none of the four cases showing extensive tumour necrosis, defined as more than $95 \%$ of tumour area, showed evidence of progression. The nine cases with necrosis mentioned in the contemporaneous pathology reports that did progress showed a variable degree of necrosis, but none showed extensive $(>95 \%)$ necrosis. All the tumours that progressed or caused death were pTNM stage 3 tumours. Thus, the fact that these tumours progressed was probably related to the higher stage at presentation, rather than to the

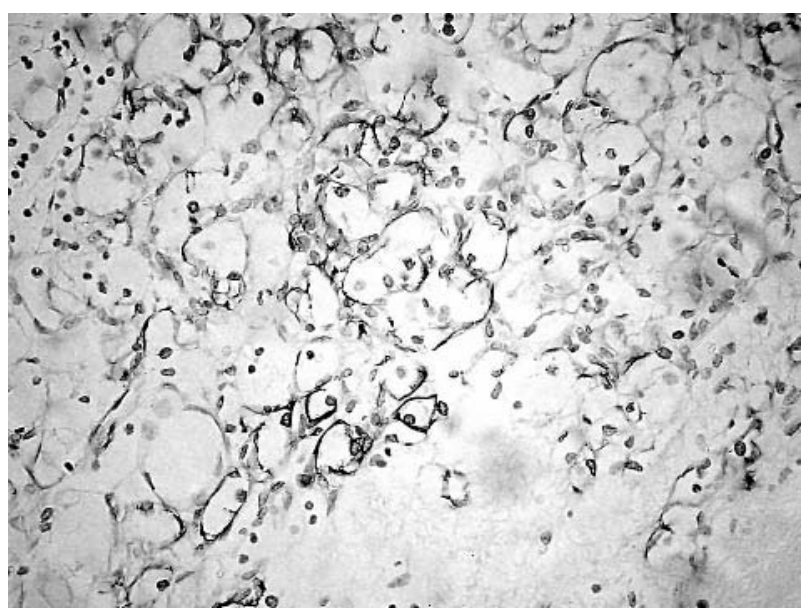

Figure 5 A high power detail of the section shown in fig 4 showing Cam 5.2 staining of a few remaining viable tumour cells within the centre of this lesion. 


\section{Take home messages}

- Extensive necrosis (>95\% necrosis) is a rare finding in renal cell carcinoma (RCC), accounting for only $1.6 \%$ of those diagnosed during eight years in this unselected hospital series

- The microscopic pattern of necrosis was typical, requiring extensive tumour sampling for definitive tumour diagnosis

- Although there were only four patients with extensive necrosis in our series, none developed recurrent or metastatic carcinoma, or died from RCC

- However, although extensive (>95\%) necrosis may imply better short term prognosis, after adjusting for tumour PTNM stage, it is probably not a prognostic variable in RCC

degree of necrosis present. The number of cases in this series is too small to perform valid statistical analyses to assess the relative prognostic effect of nuclear grade and necrosis. However, all three cases that progressed or caused death in group 2 showed nuclear grade 3 . Of the six cases that progressed in group 3, one, three, and two cases showed nuclear grades 1, 2, and 3, respectively. Overall, however, there was no clear difference in prognosis within the three necrosis subgroups if the effect of pTNM staging is taken into consideration.

RCC tumours showing extensive necrosis are rare, accounting for only $1.6 \%$ of all RCCs diagnosed during an eight year period from a non-selected population in a regional hospital. The cause of extensive necrosis in RCC is not clear. RCC is a tumour that can rarely show spontaneous regression. ${ }^{10}{ }^{15}$ Several explanations for this are possible, including autoinfarction as a result of renal vein thrombosis, coexistent severe renal artery atherosclerosis, the tumour outgrowing the vascular supply, or autoimmune mechanisms. ${ }^{16}$ None of our cases with extensive necrosis showed evidence of renal vein invasion. In fact, the five cases that did show evidence of tumour renal vein involvement were in groups 2 and 3. Similarly, none of our cases in group 1 showed evidence of severe atherosclerosis of the renal artery sufficient to cause autoinfarction. If necrosis is to be viewed as a sign of a rapidly growing tumour outgrowing its vascular supply, then one would expect extensively necrotic tumours to have a worse prognosis than usual types of RCC. This is certainly not borne out in our study. RCC carcinoma is unusual among solid tumours because of its immunogenic properties and its susceptibility to immunotherapy. ${ }^{17}$ Recent studies have highlighted the susceptibility of RCC to immunological control using allogenic peripheral blood stem cells. ${ }^{16}$ These patients had metastatic RCC refractory to conventional treatment. ${ }^{16}$ There have also been reports of spontaneous regression of metastases of RCC. ${ }^{15}$ Therefore, it is possible that RCC showing extensive necrosis may, in some cases, represent a group of tumours showing regression as a result of an autoimmune mechanism.

RCC showing extensive necrosis may cause diagnostic difficulty and such cases must be thoroughly sampled, especially around the rim of the tumour to identify small populations of viable tumour cells. ${ }^{9}$ Immunohistochemistry, particularly for cytokeratins, can often be of help in such cases.

In any prospective series of RCCs preoperative assessment using conventional or spiral computed tomography can help to assess tumour necrosis preoperatively. This was not available for this series of patients.

In summary, extensive necrosis in renal cell carcinoma may imply better short term prognosis, although after adjusting for tumour pTNM stage, very extensive (>95\%) tumour necrosis is probably not a prognostic variable in RCC. Extensive sampling of very necrotic tumours is essential to identify foci of viable tumour. The mechanism of necrosis or regression may vary from case to case and may be vascular, immunological, or arise as a result of other mechanisms.

\section{ACKNOWLEDGEMENTS}

The authors are grateful to Professor J Epstein for reviewing this manuscript.

\section{Authors' affiliations}

V Foria, T Surendra, D N Poller, Department of Pathology, Queen

Alexandra Hospital, Cosham, Portsmouth, P06 3LY, UK

\section{REFERENCES}

1 Amin MB, Tamboli P, Javidan J, et al. Prognostic impact of histologic subtyping of adult renal epithelial neoplasms: an experience of 405 cases. Am J Surg Pathol 2002;26:281-91.

2 Cheville JC, Lohse CM, Zincke $\mathrm{H}$, et al. Comparisons of outcome and prognostic features among histologic subtypes of renal cell carcinoma. Am J Surg Pathol 2003;27:612-24.

3 Ljungberg B, Alamdari FI, Stenling R, et al. Prognostic significance of the Heidelberg classification of renal cell carcinoma. Eur Urol 1999;36:565-9.

4 Lau WK, Cheville JC, Blute ML, et al. Prognostic features of pathologic stage T1 renal cell carcinoma after radical nephrectomy. Urology 2002;59:532-7.

5 Leibovich BC, Blute ML, Cheville JC, et al. Prediction of progression after radical nephrectomy for patients with clear cell renal cell carcinoma: a stratification tool for prospective clinical trials. Cancer 2003;97:1663-71.

6 Frank I, Blute ML, Cheville JC, et al. An outcome prediction model for patients with clear cell renal cell carcinoma treated with radical nephrectomy based on tumor stage, size, grade and necrosis: the SSIGN score. J Urol 2002; 168:2395-400.

7 Cheville JC, Blute ML, Zincke H, et al. Stage pTl conventional (clear cell) renal cell carcinoma: pathological features associated with cancer specific survival. J Urol 2001;166:453-6.

8 Leibovitch I, Lev R, Mor Y, et al. Extensive necrosis in renal cell carcinoma specimens: potential clinical and prognostic implications. Israel Medical Association Journal 2001;3:563-5.

9 Brinker DA, Amin MB, de Peralta-Venturina $M$, et al. Extensively necrotic cystic renal cell carcinoma: a clinicopathologic study with comparison to other cystic and necrotic renal cancers. Am J Surg Pathol 2000;24:988-95.

10 Hamid Y, Poller DN. Spontaneous regression of renal cell carcinoma: a piffall in diagnosis of renal lesions. J Clin Pathol 1998;51:334-6.

11 Fuhrman SA, Lasky LC, Limas C. Prognostic significance of morphologic parameters in renal cell carcinoma. Am J Surg Pathol 1982;6:655-63.

12 Sobin LH, Wittekind C. TNM classification of malignant tumours, 5 th ed. New York: John Wiley, 1997.

13 Amtrup F, Hansen JB, Thybo E. Prognosis in renal cell carcinoma evaluated from histological criteria. Scand J Urol Nephrol 1974;8:198-202.

14 Hartman DS, Davis CJ Jr, Johns T, et al. Cystic renal cell carcinoma. Urology 1986;28:145-53.

15 Fairlamb D. Spontaneous regression of metastases of renal cell cancer: a report of two cases including the first recorded regression following irradiation of a dominant metastasis and review of the world literature. Cancer 1981;47:2102-6.

16 Childs R, Chernoff A, Contentin N, et al. Regression of metastatic renal cell carcinoma after nonmyeloablative allogenic peripheral blood stem cell transplantation. New Engl J Med 2000;343:750-8

17 Figlin RA, Pierce WC, Kaboo R, et al. Treatment of metastatic renal cell carcinoma with nephrectomy, interleukin 2 and cytokine primed or CD $8(+)$ selected tumor infiltrating lymphocytes from primary tumor. $J$ Urol 1997;158:740-5. 